\title{
Soft Skills and Psychosocial Counselling in COVID-19 Times: the Changing Tactics
}

\author{
Kritant Bhushan $^{1} \cdot$ Sanat Khanna $^{1} \cdot$ Mansi Luthra Sharma ${ }^{1} \cdot$ Prathiba Rai $^{1}$
}

Received: 30 June 2021 / Accepted: 25 August 2021/Published online: 14 September 2021

(C) The Association of Oral and Maxillofacial Surgeons of India 2021

\begin{abstract}
Introduction As the COVID-19 pandemic is increasingly griping the world, it starts effecting the psychosocial health of the patients, health care workers, frontline workers and their dependents. The entire landscape of existing patient psychological counselling and management needs to be changed to avoid fear and misinformation about COVID19.

Material and methods In this article, we have tried to summarize the rules, regulations and protocols in accordance with government guidelines along with practical implementation of counselling in management of psychological health condition of patients and frontline workers. Conclusion If used wisely and as per recommendations, psychosocial counselling is a very useful tool in the present COVID-19 pandemic, to avoid spread of misinformation, fear and grief about the disease which creates mental health issue and anxiety among close contacts as well as health care workers.
\end{abstract}

Keywords COVID-19 pandemic · Psychosocial counselling $\cdot$ Mental health · Frontline workers

Kritant Bhushan

Kritant83@gmail.com

Mansi Luthra Sharma

drmansi17@gmail.com

1 Govt Hospital, Shimla, India

\section{Introduction}

COVID-19, a communicable disease, has instilled fears in the minds of the community because of severe morbidity, mortality and efficacy of high transmission. The constant fears of getting the infection and passing the infection to friends, families and co-workers make one culprit of spreading infection that ultimately lead to the development of mental stress [1]. The fears of contracting the illness are also frequent and range from misinterpreting every fever or cough as a COVID-19 infection, getting test themselves without need of any, to hoarding medications despite there not being indications for their generalized use, also what type of mask, what distances to maintain, what surfaces need disinfection. Among the population, there are certain worries of loss of jobs and economic slowdown during the pandemic. There are lot of other issues which leads to a cycle of concern, worry, and distress. Some of these fears and behaviors are realistic, while many are just borne out of lack of knowledge, rumors and misinformation. This has created an unprecedented mental health challenge [1,2]. Frontline personnel need to be trained in simple and emerging evidence-based strategies of assessment and management, also we need to engage with non-psychiatric medical professionals to facilitate the psychiatric management of patients with COVID-19 infection and comorbid physical illnesses.

\section{Basic Principles of Psychosocial Counselling}

Five essential principles of psychosocial care, as depicted by Hobfoll; these are Sense of safety, Sense of calmness, Building hopes, Self and collective efficacy, Connectedness $[2,3]$. 


\section{Goals of Counselling}

Encouraging safety, health and hygiene, reducing immediate distress and anxiety, normalize the worry and developing healthy ways of addressing worry. Help individuals cope better with their life challenges and suggest some appropriate ways to enhance coping and generate a sense of realistic hope [3].

\section{Counselling Skills}

Effective counselling would need the following skills,

1. Attending the patients cautiously and follow COVID appropriate behavior while attending patients and attendants.

2. Active and carefully listening and must have nonjudgmental attitude.

3. Demonstrating empathy and provide emotional support and assuring confidentiality.

4. Providing basic facts, information and reliable resources to the clients regarding their concerns and clarify the myths about COVID-19.

5. Enhance self-esteem and reduce stigma and assessing risks if client experiencing any thoughts about harming themselves and crisis management if required.

\section{Qualities of an Effective Counsellor}

He should be warm, flexible in approach, good communication skills, updating oneself regularly, always nonjudgmental and easily approachable through telemedicine $[3,4]$.

\section{General Advice for Coping}

Maintain healthy lifestyle, get enough sleep, maintain good sleep pattern, eat healthy and balanced diet. Involve in regular yoga/exercises, avoid drugs/alcohols. Maintain sense of high hope, positive approach, stay away from misinformation about COVID and negative news. Communicate to patients that health authorities are genuinely helping them, positive effects of quarantine, lockdown and vaccination [4].

\section{Use of Digital Platforms in Training and Support- COVID-19}

In the current scenario of COVID-19 pandemic, telemedicine is emerging as a key technology for efficient communication and sustainable solution to provide essential health care services. The steps involved are given in Fig. 1.

\section{Psychosocial Counselling for COVID Positive Patients}

People diagnosed with COVID-19 may experience a range of distress during their course of illness like shock, fear, denial to anger, irritability, frustration and many more, sometimes, distress becomes so overwhelming for few that it has a deleterious impact on the patients mental health that ultimately impeding recovery in many cases. Thus, an urgent need for providing psychosocial care to the patients to optimize their mental health and well-being [4, 5].

Steps of counselling: For COVID-19 patients, disease is divided into three phases and counselling to be done in phase manner as the disease progress which is clearly understood by Fig. 2 below.

The aim of counsellor is to support the recovery of the patient in such a way that their immediate distress is normalized and managed, their specific concerns are addressed, coping skills are enhanced, and hope perspective is instilled. The counsellors must use the SPARC method, which stands for stabilization, psychoeducation, addressing adjustment issues, recovery and coping skill enhancement [5].

\section{Psychosocial Counselling for Family Members/ Primary Contact of COVID Positive Patients}

As per MOHFW, primary contact can be any person who is involved in any of the following: providing direct care without proper personal protective equipment (PPE) for COVID-19 patients and staying in the same close environment as a COVID-19 patient (including workplace, classroom, household, gatherings) $[4,5]$. Travelling together nearby $(1 \mathrm{~m})$ with the asymptomatic person who later tested positive for COVID-19. Based on risk, there are two types of contacts.

High-Risk Contact: which has direct physical contact with the patient and their fluids including physical examination without PPE, anyone nearby (within $3 \mathrm{ft}$ ) of the confirmed case without precautions.

Low-Risk Contact: Shared the same space (Same class for school/worked in the same room/similar and not having a high-risk exposure to a confirmed or suspect case of COVID-19).

Issues faced by COVID + family member (CFM) or primary contact $(C P C)$ : Various issues faced by family members and primary contact including pregnant ladies, children, elderly people, any mentally or physical disabled person at home are [4-6] as follows: 
Fig. 1 Shows steps involve in digital training

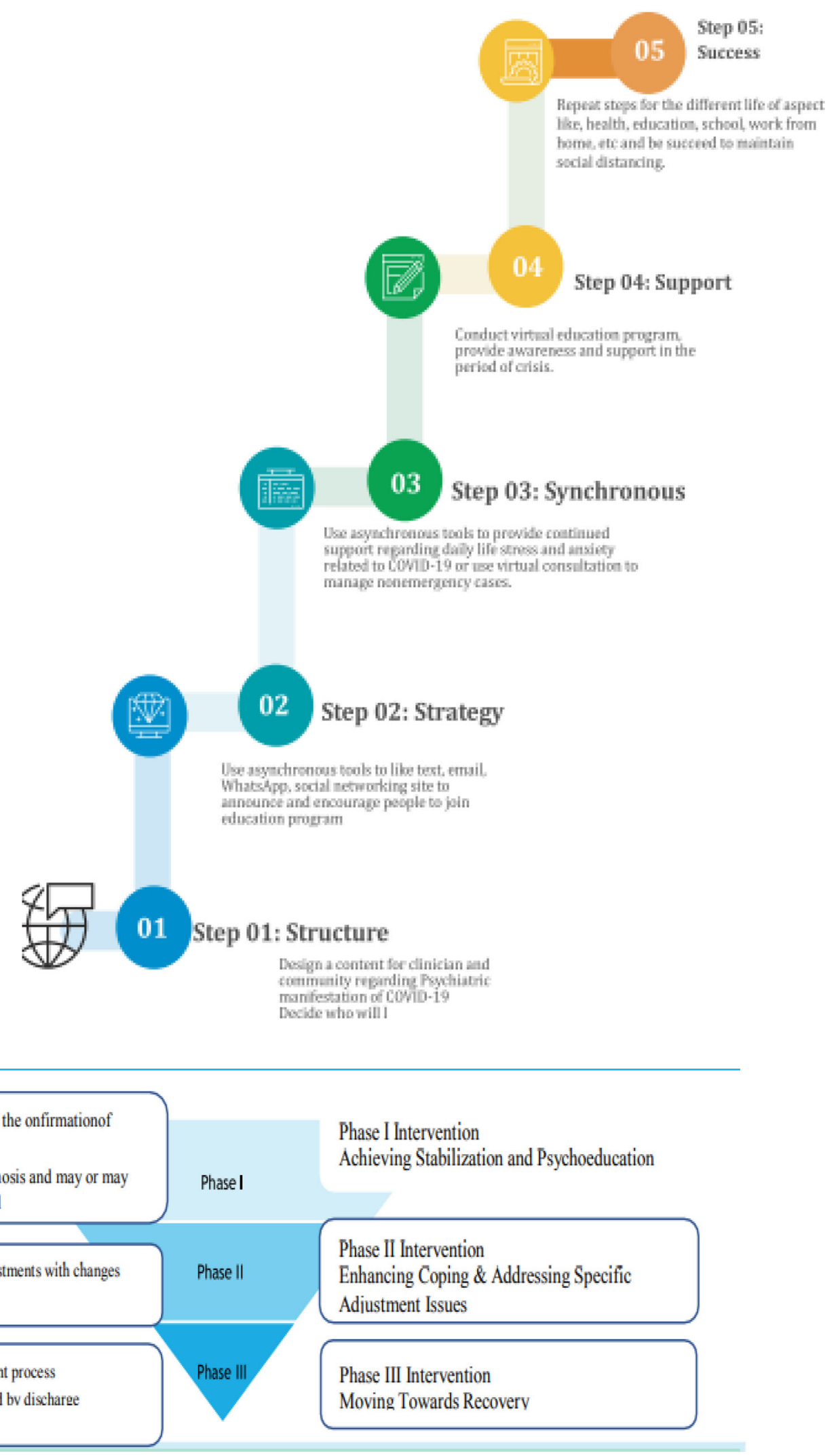

Fig. 2 Shows different phase intervention 


\section{Psychosocial Health Issues in Quarantine/Isolation:}

These include

1. Health-related depression anxiety like fear of turning positive on testing, stigma and contracting a severe and possibly life-threatening illness.

2. Low mood, fear, nervousness, irritability, anger, frustration, boredom, emotional exhaustion, feeling stressed, numbness, and insomnia.

3. Acute stress reaction and post-traumatic stress disorder (PTSD).

\section{Management}

Validation-Acknowledge the feeling of boredom, loneliness, feeling sad, stressed, confused, loss of personal freedom and guilt. Provide access to family and friends through mobile phones. Provide relevant and correct information as possible about the infection and rationale behind the quarantine $[6,7]$. Avoid speculation and break the chain of rumor during the hospital quarantine. Individual to remain physically active and ensure a balanced diet to keep themselves fit and calm [8].

\section{Psychosocial Health Issues in Children and Adolescents}

Children are confined to the home and in some situations may be separated from the parents because either they are quarantined, or their parents are quarantined. This leads to range of psychological issues such as anxiety, fear, worry, depression, difficulty sleeping, and loss of appetite, acute stress disorder, PTSD and grief in many children [8, 9]. Watch for excessive crying or irritation, worry or sadness, unhealthy eating or sleeping habits, difficulties with attention and concentration [9].

\section{Management}

Explain your child about COVID-19 facts in easy and simple manner, limit your family's exposure to negative news coverage of the COVID-19, including social media, try to keep up with regular routines, learn new activities, get plenty of sleep, exercise, and eat well. Connect with your friends and family members [8, 9].

\section{Psychosocial Health in Older Adults}

Older adults are consistently reported to be more vulnerable to COVID-19. According to the Centre for disease control and Prevention (CDC), older adults with COVID19 are more likely to be hospitalized (31-59\%) and die of it
(4-11\%). This risk is higher in older age group above 80 years [10, 11].

Issues faced by older adult are as follows: high death rates due to multiple old age problem combined with COVID-19 complications, more stress and difficulty in accessing essential services due to the restrictions enforced to maintain the social distancing to prevent the spread of COVID-19, guilt feeling to depends on other, social isolation, less knowledge to use new technology, living alone or in old age home setup leads to more stress and anxiety which cause more complications along with COVID-19, old age multiple problems with regular visit to hospitals make them more vulnerable to COVID infection [11, 12].

\section{Management}

Defer unnecessary visit to hospitals, try to take telemedicine consultation for their underlying health aliments. Utilizing community health workers or trained social workers for the screening of older adults at old age homes/ assisted living facilities. Maintaining a routine, physical exercise, having healthy nutritious diet, is highly recommended. Older adults need reassurance that most of the mental health issues experienced in these situations are normal reactions to abnormal stress $[11,12]$.

\section{Psychosocial Health Issues among Pregnant and Postpartum}

Positive mental health is particularly important during pregnancy and postpartum. In the current period of COVID-19 pandemic, pregnant women and COVID-19 related worries are $[13,14]$ as follows:

1. What precaution can I take to prevent infection?

2. How this virus will effect my unborn baby?

3. Is it safe to go to the hospital for antenatal check-ups or scans?

4. Is excessive use of hand sanitizer safe during pregnancy?

5. What if I get an infection, will I transmit it to my baby?

6. Is it safe to breastfeed my baby?

7. Is it safe to immunize my child?

\section{Management}

Minimize watching, reading or listening to news about COVID-19 that makes one anxious or distressed. Avoid going to hospital for antenatal and prenatal checkup, advice and prefer telemedicine. Physical health during pregnancy can be sustained by maintaining an adequate diet which includes green leafy vegetables, protein, and 
carbohydrates. Continue iron and folic acid tablets prescribed by doctor [13, 14].

Currently, no research has shown that COVID spread to unborn child through mother. Breastfeed women must follow precautions to limit viral spread to the baby which includes hand washing before touching the baby, breast pump or bottles, Try and avoid coughing or sneezing on your baby while feeding at the breast, Consider wearing a face mask while breastfeeding [14].

\section{Psychosocial Health Issues in ICU Patients}

The nature of COVID-19 isolation procedures destabilizes the emotional balance, generating feelings of insecurity, disbelief, helplessness, and hopelessness. This provides a fertile ground for a variety of psychiatric manifestations to develop, in an ICU setting $[15,16]$.

\section{Delirium (ICU psychosis)}

Mechanical ventilation is required in a small proportion of COVID-19 patients, which sometime especially in old age people cause anxiety, sleep disturbance, depression, delirium, communication problems, pain, fear of being dependent on the machine for breathing, rapid fluctuation in emotions like sudden unprovoked irritability, crying spells, fluctuation in levels of consciousness, visual hallucinations [16]

If a COVID-19 infected patient becomes disruptive then, the management of such a disruptive behavior is of utmost importance as it could result in a break in the isolation protocol and may place health care professionals at higher risk of exposure to infection.

\section{Management}

Enhance sensory efficacy (e.g., encouraging patient to use their glasses or hearing aids), promote sleep, adequate and appropriate pain management, preventing complications of immobility (bed sores), optimization of physiological parameters (e.g., electrolytes, hydration), and foster physical therapy/early mobilization $[16,17]$.

\section{Psychosocial Issues of Frontline Personnel}

COVID-19 presents the front line of a new uncertain battle for the human race. Thousands of health care personnel and government officials are working round the clock to confront it. Issues faced by them are as follows: long working hours, worry about risk to self and family, concerns about inadequate personal protection materials, separation from family/loved ones, staff shortage, and often experience stigmatizing attitudes from the general public [17-19].
Management

1. Self-care: All personnel in the front line should be made aware of the principles of self-care which emotional, physical, relational, and spiritual/religious wellness [20].

2. Team Leaders/Supervisors: Ensure that juniors with limited experience work with their senior colleagues, Ensure staff rotation from jobs of higher stress to lower stress and vice versa, Duty/shift breaks/holidays to be agreed within the team and ensured as far as possible, Ensure good quality communication with accurate information updates, Have regular team meetings which helps to develop a 'bond' and to also sort out issues that may emerge because of working in stressful situation. Buddy system to be introduce If a team member is experiencing mental health difficulties. The 'buddy' can be a senior colleague/workmate, who may be expected to talk and listen and provide common sense suggestions for mental health care $[17,20]$.

\section{Psychosocial Issues of Vaccination}

India being a developing nation with large no of population still resides in villages and literacy rate is low certain myths are spreading about vaccine like, the COVID-19 vaccine is unsafe because it was developed so quickly, vaccine will alter my DNA, vaccine includes a tracking device, causes infertility in women and men, Once I receive the COVID-19 vaccine, I no longer need to wear a mask etc.

\section{Management}

Provide correct information about the vaccine in simple and effective way so that the myth associated with vaccine is duly declined and vaccine must be taken by all.

\section{Conclusion}

India currently has approved two vaccines to prevent coronavirus disease, i.e., Covaxin and Covishield. Apart from vaccination, the best way to prevent illness is to avoid being exposed to this virus. The fear, spreading misinformation and grief about the disease create mental health issues and anxiety among the close contacts. Hence, systematically adhering to protective measures shall mitigate fear and anxiety. Understanding the complexities of anxiety and grief can be important for supporting patients, families, colleagues, and ourselves. It is important to emphasize that the techniques mentioned in the present 
guidelines under each phase constitute the Intervention's core elements; the module does not follow a rigid sequential approach. Thus, health care professionals are encouraged to modify, adapt and tailor their preset intervention approach based on guidelines described below to fit the ecology of the culture, place, type and severity of distress of the patients with COVID-19.

\section{Funding None.}

\section{Declarations}

Conflict of interest There are no conflicts of interest.

\section{References}

1. Brooks SK, Webster RK, Smith LE, Woodland L, Wessely S, Greenberg $\mathrm{N}$ et al (2020) The psychological impact of quarantine and how to reduce it: rapid review of the evidence. Lancet 395:912-920

2. Huremović D (ed) (2019) Psychiatry of pandemics: a mental health response to infection outbreak. Springer International Publishing, Cham

3. Johal SS (2009) Psychosocial impacts of quarantine during disease outbreaks and interventions that may help to relieve strain. New Zealand Med J 122:53-58

4. Mental Health in the times of COVID-19 Pandemic Guidance for General Medical and Specialised Mental Health Care Settings. National Institute Of Mental Health \& Neurosciences, Bengaluru, India. Accessed 13 April-2020

5. Li YC, Bai WZ, Hashikawa T (2020) The neuroinvasive potential of SARS-CoV2 may play a role in the respiratory failure of COVID-19 patients. J Med Virol. https://doi.org/10.1002/jmv. 25728. [Epub ahead of print]

6. Mental health and psychosocial considerations during the COVID-19 outbreak. World Health Organisation. Available at https://apps.who.int/iris/bitstream/handle/10665/331490/WHO2019-th nCoV-MentalHealth-2020.1-eng.pdf. Acessed 04 April 2020.

7. Dalton L, Rapa E, Stein A (2020) Protecting the psychological health of children through effective communication about COVID-19. Lancet Child Adolesc Health 4(5):346-347. https:// doi.org/10.1016/S2352-4642(20)30097-3
8. Wang G, Zhang Y, Zhao J, Zhang JJF (2020) Mitigate the effects of home confinement on children during the COVID-19 outbreak. Lancet 395(10228):945-947

9. Armitage R, Nellums LB (2020) COVID-19 and the consequences of isolating the elderly. Lancet Public Health 2667(20):30061. https://doi.org/10.1016/S2468-2667(20)30061$\mathrm{X}$

10. World Health Organization (2020) Mental health and psychosocial considerations during the COVID19 outbreak. Retrieved from https://www.who.int/docs/default-source/coronaviruse/men talhealth-considerations.pdf

11. Yang Y, Li W, Zhang Q, Zhang L, Cheung T, Xiang YT (2020) Mental health services for older adults in China during the COVID-19 outbreak. Lancet Psychiatry. https://doi.org/10.1016/ S2215-0366(20)30079-1

12. Yao H, Chen JH, Xu YF (2020) Patients with mental health disorders in the COVID-19 epidemic. Lancet Psychiatry 7(4):e21. https://doi.org/10.1016/S2215-0366(20)30090-0

13. Coronavirus (COVID-19). Infection in Pregnancy Information for Healthcare Professionals. Version 6 Published Friday 3 April 2020.

14. Perinatal Psychiatry NIMHANS - Home. https://www.perina talpsynimhans.org/. Accessed April 4, 2020.

15. Levenson, JL (2019) American Psychiatric Association. Textbook of Psychosomatic Medicine and Consultation-Liaison Psychiatry, 3rd edn.

16. Arumugam S, El-Menyar A, Al-Hassani A, Strandvik G, Asim M, Mekkodithal A et al (2017) Delirium in the intensive care unit. J Emergencies Trauma Shock. https://doi.org/10.4103/09742700.199520

17. Greenberg N, Docherty, Gnanapragasam S, Wessely S (2020) Managing mental health challenges faced by healthcare workers during COVID-19 pandemic. British Med J, 368.

18. World Health Organization (2020) Mental health and psychosocial considerations during the COVID-19 outbreak, 18 March 2020 (No. WHO/2019-nCoV/Mental Health/2020.1). World Health Organization.

19. Scarella TM, Boland RJ, Barsky AJ (2019) Illness anxiety disorder: psychopathology, epidemiology, clinical characteristics, and treatment. Psychosomatic Med 81(5):398-407

20. Chen Q, Liang M, Li Y, Guo J, Fei D, Wang L et al (2020) Mental health care for medical staff in China during the COVID19 outbreak. Lancet Psychiatry 7(4):e15-e16

Publisher's Note Springer Nature remains neutral with regard to jurisdictional claims in published maps and institutional affiliations. 